\title{
INIBIÇÃO DA AÇÃO DO ETILENO NA CONSERVAÇÃO DE CAQUIS (Diospyrus kaki L.) 'FUYU'
}

\author{
CASIANE SALETE TIBOLA², LUCIANO LUCCHETTA ${ }^{3}$, MÁRCIO ROGGIA ZANUZO ${ }^{3}$, PAULO ROBERTO DA SILVA ${ }^{4}$, \\ VALDECIR CARLOS FERRI ${ }^{5}$, CÉSAR VALMOR ROMBALDI ${ }^{4}$
}

\begin{abstract}
RESUMO - Com o objetivo de ampliar o período de conservação e de comercialização do caqui 'Fuyu', foram testadas alternativas para a manutenção da qualidade dos frutos. Realizaram-se três experimentos: 1 ) os frutos foram armazenados na temperatura de $18 \pm 3^{\circ} \mathrm{Ce} \mathrm{UR} \mathrm{de} 80 \pm 5 \%$, como ou sem aplicação de 1-MCP, avaliados na colheita aos 7 e aos 14 dias; 2) os frutos foram conservados na temperatura de $0 \pm 1,0^{\circ} \mathrm{C}$ e UR de $95 \pm 5 \%$, com ou sem aplicação de 1-MCP, avaliados na colheita e aos $45+3$ dias de comercialização simulada, e 3) os frutos foram armazenados em atmosfera modificada (AM) com filmes de polietileno de baixa densidade (PEBD) de $80 \mu \mathrm{m}$ de espessura e em temperatura de $0 \pm 1,0^{\circ} \mathrm{Ce} \mathrm{UR}$ de $95 \pm 5 \%$, com ou sem aplicação de 1-MCP, avaliados na colheita, aos $48+4$ dias e aos $48+10$ dias de comercialização simulada (CS) na temperatura de $18^{\circ} \mathrm{C}$. A concentração de 1-MCP utilizada nos tratamentos específicos foi de 500ppb/2h. As principais análises realizadas compreenderam: firmeza da polpa (FP), cor e escurecimento de epiderme (EE). Os caquis do experimento 2 perderam a qualidade de comercialização antes de alcançarem os 30 dias de estocagem. O uso de AM associada à refrigeração manteve frutos com FP acima de $40 \mathrm{~N}$, além de prevenir a ocorrência de EE, após 48 dias de armazenamento mais 10 dias de CS. A obtenção destes índices proporcionou a manutenção da qualidade dos caquis por 48 dias de câmara mais 10 dias de CS no ar ambiente $\left(18^{\circ} \mathrm{C}\right)$.
\end{abstract}

Termos de indexação: 1-metilciclopropeno, escurecimento de epiderme, comercialização simulada, Diospyrus kaki.

\section{ETHYLENE INHIBITOR ACTION IN THE STORAGE OF PERSIMMON FRUITS (Diospyrus kaki L.) 'Fuyu'}

ABSTRACT: With the objective of to extend the conservation period and commercialization of the 'Fuyu' persimmon fruit, alternatives to mantain fruits quality were tested. Tree experiments were accomplished to develop the work: 1) storage at the ambient temperature (AT): Temperature (T) of $18 \pm 3^{\circ} \mathrm{C}$ and relative humidity (RH) of $80 \pm 5 \%$, with or without application of 1 -MCP and evaluated in the crop, at 7 and 14 days; 2 ): cold storaged (CS): $\mathrm{T}$ of $0 \pm 1,0^{\circ} \mathrm{C}$ and $\mathrm{RH}$ of $95 \pm 5 \%$, with or without application of 1-MCP, appraised in the crop and $45+3$ days of simulated commercialization; and 3) CS and modified atmosphere (MA) with films of polyethylene of low density (PELD) with $80 \mathrm{~mm}$ of thickness: T of $0 \pm 1,0^{\circ} \mathrm{C}$ and RH of $95 \pm 5 \%$, with or without application of 1-MCP, appraised in the crop, 48 of CS and 10 days of simulated commercialization. The concentration of 1-MCP used in the specific treatments was of 500ppb/2h. The main physiochemical analyses accomplished were: pulp firmness (PF), color and epidermis browning (EB). The persimmon fruits of the experiment 2, maintained in CS, lost the commercialization quality before reaching 30 days of storage. The use of MA associated to CS, maintained the fruits with PF above $40 \mathrm{~N}$, besides preventing the occurrence of EB. The yielded results showed that the maintenance of persimmon fruits quality can be kept for 48 days at CS and more 10 days of simulated commercialization at the environment temperature.

Index terms: 1-metilciclopropene, epidermis browning, simulated commercialization, Diospyrus kaki .

\section{INTRODUÇÃO}

O 'Fuyu' é a cultivar de caquizeiro mais plantada no Brasil (João et al., 2002), produz frutos sem sementes, de polpa brancoamarelada, com sabor adocicado, sem adstringência, com baixa acidez e epiderme amarelo-avermelhada (Ferri et al., 2002b).

O período de colheita e oferta desta fruta ainda é relativamente curto, aproximadamente 60 dias, nos meses de maio e junho. Neste período, a oferta é elevada e, conseqüentemente, os preços de comercialização são reduzidos e as perdas pós-colheita elevadas (Ferri et al., 2002a; Donazzolo \& Brackmann, 2002).

O caqui 'Fuyu' caracteriza-se por apresentar um comportamento climatérico (Girardi et al., 2003) e tem alta sensibilidade ao etileno, o que proporciona rápida evolução da coloração da epiderme e perda de firmeza da polpa (Gorini \& Testoni, 1988). Além disso, os frutos dessa cultivar são sensíveis à ocorrência de escurecimento epidérmico, que é, juntamente com o amolecimento, a principal causa de perda de caquis armazenados (Donazzolo \& Brackmann, 2002). Embora as causas desse distúrbio não tenham sido elucidadas, vários autores emitem hipóteses relacionando com fatores pré-colheita, como a sanidade do pomar, desequilíbrio nutricional e ponto de maturação (Brackmann \& Donazzolo, 2000), elevada umidade relativa no período de maturação (Gonçalves, 2003) e senescência dos frutos (Brackmann et al., 1997).

Para prolongar o período de conservação de caqui 'Fuyu', três alternativas principais têm sido recomendadas: a) armazenamento em ar refrigerado (AR) (Brackmann et al., 1997); b) armazenamento em atmosfera modificada (AM) (Antoniolli et al., 2003), e c) armazenamento em atmosfera controlada (AC) (Brackmann et al., 2003).

Para melhorar a conservabilidade do 'Fuyu' e prolongar o período de comercialização, os principais resultados obtidos apontam para o uso de AM (Antoniolli et al., 2003) e AC (Brackmann et al., 2003). Em AM, sugere-se o uso de embalagens de polietileno de 0,05 a 0,06 mm de espessura e temperatura de armazenamento de $0^{\circ} \mathrm{C}$ (Antoniolli et al., 2003). Em AC, recomenda-se a concentração de $8 \mathrm{KPa}$ de $\mathrm{CO}_{2}$ e de $2 \mathrm{KPa}$ de $\mathrm{O}_{2}$, na temperatura de $-0,5^{\circ} \mathrm{Ca} 0{ }^{\circ} \mathrm{C}$ (Brackmann \& Saquet, 1995).

Por se tratar de uma fruta sensível ao etileno, a redução da produção e/ou da ação deste hormônio pode prolongar a vida de prateleira dessa fruta (Sisler, 1991). Os benefícios dessa ação podem ser obtidos com o uso de AR, AM e AC, associados ou não a adsorvedores de etileno (Neves et al., 2003). A síntese da molécula de 1Metilciclopropeno (1-MCP), que é um potente inibidor da ação do etileno, tem sido uma nova alternativa para prolongar o armazenamento de frutos (Corrent, 2003). Portanto o emprego desse inibidor pode prolongar o período seguro de armazenamento, tanto em temperatura ambiente quanto em AR e AM.

Neste contexto, o trabalho tem por objetivo verificar o efeito de redução da ação do etileno, com o emprego de 1-MCP, na conservação de caquis 'Fuyu', armazenados à temperatura ambiente, sob refrigeração e em AM.

\footnotetext{
${ }_{1}^{1}$ (Trabalho 074/2004). Recebido: 16/06/2004. Aceito para publicação: 06/04/2005. Projeto realizado com apoio financeiro da FAPERGS e Kiwis'trin Ltda.

${ }^{2}$ Eng. Agra . Doutoranda do PPGA, Área de Concentração Fruticultura de Clima Temperado - UFPel/FAEM, Cx. P: 354, CEP: 96010-900. Pelotas - RS. casiane@ufpel.tche.br

${ }^{3}$ Eng. Agr ${ }^{\circ}$. M.Sc. Doutorando do PPGCTA - UFPel/FAEM. lucianolucchetta@yahoo.com.br / zanuzo@ yahoo.com.br

${ }^{4}$ Acadêmico de Agronomia da UFPel/FAEM. paulopalmitinho@yahoo.com.br

${ }_{5}^{5}$ Eng. Agr ${ }^{\circ}$. Dr. Professor do Departamento de Ciência e Tecnologia Agroindustrial da UFPel/FAEM. leferri@ufpel.tche.br
} 
TABELA 1 - Qualidade de caquis 'Fuyu', armazenados em temperatura ambiente $\left(18 \pm 3^{\circ} \mathrm{C}\right)$, avaliados na colheita, aos 7 e aos 14 dias de conservação.

\begin{tabular}{lllll}
\hline Parâmetros & Aplicação de & Períodos de análise & & \\
\cline { 2 - 4 } & $1-\mathrm{MCP}$ & Colheita & $48+4$ dias & $48+10$ dias \\
\hline Firmeza de Polpa (N) & Controle & $82,6 \mathrm{aA}$ & $61,7 \mathrm{aA}$ & $48,1 \mathrm{aB}$ \\
& $1-\mathrm{MCP}$ & $82,6 \mathrm{aA}$ & $62,1 \mathrm{aA}$ & $65,7 \mathrm{bA}$ \\
Cor "a" & Controle & $34,6 \mathrm{aA}$ & $35,1 \mathrm{aA}$ & $36,8 \mathrm{aA}$ \\
& $1-\mathrm{MCP}$ & $34,6 \mathrm{aA}$ & $34,9 \mathrm{aA}$ & $43,0 \mathrm{aA}$ \\
Cor "b" & Controle & $49,7 \mathrm{aA}$ & $50,4 \mathrm{aA}$ & $47,4 \mathrm{aA}$ \\
& $1-\mathrm{MCP}$ & $49,7 \mathrm{aA}$ & $50,7 \mathrm{aA}$ & $52,0 \mathrm{aA}$ \\
Cor "L" & Controle & $56,3 \mathrm{aA}$ & $56,4 \mathrm{aA}$ & $51,2 \mathrm{aB}$ \\
& $1-\mathrm{MCP}$ & $56,3 \mathrm{aA}$ & $55,1 \mathrm{aA}$ & $54,4 \mathrm{aA}$ \\
Ângulo Hue & Controle & $54,6 \mathrm{aA}$ & $55,2 \mathrm{aA}$ & $51,5 \mathrm{aA}$ \\
& $1-\mathrm{MCP}$ & $54,6 \mathrm{aA}$ & $50,4 \mathrm{aA}$ & 0 \\
Escurecimento da epiderme (\%) & Controle & 0 & 0 & 0
\end{tabular}

*Médias seguidas pela mesma letra, minúscula quando na mesma coluna e maiúscula entre colunas, não diferem estatisticamente, pelo teste de Tukey, a 5\% de probabilidade, para cada parâmetro avaliado.

\section{MATERIAL E MÉTODOS}

Foram utilizados frutos de caqui 'Fuyu', safra 2002-2003, provenientes de pomar comercial localizado no município de FarroupilhaRS, de propriedade da Empresa Kiwis'trin Ltda.

Os caquis foram colhidos em 23-05-2003, quando a coloração da casca apresentava-se alaranjado-avermelhada, com valores de $L^{*} . a^{*} . b^{*}$ de 56,30; 34,69 e 49,07, respectivamente, e ângulo Hue: 54,74. O teor de sólidos solúveis totais foi de $14,7^{\circ} \mathrm{Brix}$, a acidez total titulável de $0,793 \mathrm{cmol}$ de ácido málico $100 \mathrm{~mL} \cdot \mathrm{suco}^{-1}$, a firmeza de polpa média de $82,6 \mathrm{~N}$ e o peso médio dos frutos foi de $327,5 \mathrm{~g}$. O período decorrido entre a colheita e o armazenamento foi de 6 horas.

Para testar a hipótese, realizaram-se três experimentos:

1) Armazenamento em temperatura de $18 \pm 3^{\circ} \mathrm{C}$ e umidade Relativa (UR) de $80 \pm 5 \%$, com e sem aplicação de 1-MCP. Esse tratamento foi desenvolvido em temperatura ambiente (TA).

2) Armazenamento em ar refrigerado (AR), a $0 \pm 1,0^{\circ} \mathrm{C}$ e UR de $95 \pm 5 \%$, com e sem aplicação de 1-MCP.

3) Armazenamento em atmosfera modificada (AM), a $0 \pm 1,0^{\circ} \mathrm{C}$ e UR de $95 \pm 5 \%$, com e sem aplicação de 1-MCP. Utilizaram-se filmes de polietileno de baixa densidade (PEBD) de $80 \mu \mathrm{m}$ de espessura em ambos os lados (Canguru Embalagens Ltda, Pelotas-RS). Previamente à embalagem, os caquis foram pré-resfriados através da circulação de ar frio, até atingirem a temperatura de polpa de aproximadamente $4^{\circ} \mathrm{C}$.

O 1-MCP aplicado na concentração de 500 ppb, por um período de 2 horas, à temperatura de $20^{\circ} \mathrm{C}$ e UR de $85 \%$, em câmara fechada.

No experimento $1 \mathrm{em} \mathrm{TA}$, metade do lote de frutos foi submetida à aplicação de 1-MCP, e o restante do lote não foi tratado. Cada unidade experimental foi caracterizada por uma bandeja com seis frutos, com três repetições. As avaliações foram realizadas por ocasião da colheita, aos 7 e aos 14 dias de armazenamento.

Para o experimento 2, em AR, metade do lote de frutas foi submetida à aplicação de 1-MCP, e o restante não foi tratado. Foram utilizadas três repetições, com seis frutas cada, sendo que as avaliações foram realizadas na colheita e após 45 dias de armazenamento, +3 dias a $18 \pm 3^{\circ} \mathrm{Ce} 85 \pm 3 \%$ de UR.

No experimento $3(\mathrm{AR}+\mathrm{AM})$, metade do lote de frutas foi submetida à aplicação de 1-MCP, e o restante não foi tratado. Foram utilizadas três repetições com seis frutas cada, sendo que as avaliações foram realizadas na colheita e aos 48 dias de câmara, mais 4 e 10 dias a 18 $\pm 3^{\circ} \mathrm{C}$ e $85 \pm 3 \%$ de UR. Para o estudo de vida de prateleira, os frutos foram retirados das embalagens.

As principais análises realizadas incluíram a determinação de: 1) firmeza de polpa com penetrômetro manual de ponteira de $11 \mathrm{~mm}$, com resultados expressos em Newton $(\mathrm{N})$; 2) coloração, com um colorímetro portátil tri-estímulo Minolta modelo CR-300, através da escala $L^{*} . a^{*} . b^{*}$ e ângulo Hue. Os valores de L indicam a claridade e variam de 100 (branco) a 0 (preto); a coordenada e a abscissa b indicam a direção da cor: -a é a direção do verde e +a direção do vermelho; -b direção do azul e +b direção do amarelo. $\mathrm{O}$ ângulo Hue é definido como iniciando no eixo +a e é expresso em graus, sendo que $0^{\circ}$ corresponde a $+\mathrm{a}$ (vermelho), $90^{\circ}$ corresponde $\mathrm{a}+\mathrm{b}$ (amarelo), $180^{\circ}$ corresponde $\mathrm{a}-\mathrm{a}$ (verde) e $270^{\circ}$ corresponde $\mathrm{a}-\mathrm{b}$ (azul). Os valores de Hue foram obtidos relacionandose os valores de a e b (Hue $\left.=\operatorname{tg}^{-1} b / a\right)$, sendo que esta é a variável que melhor representa a evolução de coloração da epiderme de caquis; 3 ) ocorrência de escurecimento da epiderme (EE): quantificou-se visualmente através de notas atribuídas em escala: (0) para frutos com 0 a $19 \%$ de escurecimento; (1) para 19,1 a 50\%, e (2) acima de 50,1\% da epiderme escurecida. Para a interpretação dos resultados, realizou-se análise da variância, utilizando-se das médias para os fatores tratamentos e período de avaliação.

\section{RESULTADOS E DISCUSSÃO}

Efeito da inibição de etileno em Temperatura Ambiente (TA)

A firmeza de polpa (FP) manteve-se maior em caquis tratados com 1-MCP (47,96 N) do que nos não-tratados (32,6 N) (Tabela 1). Porém, em ambos os casos, a FP ainda foi elevada, permitindo a embalagem, o transporte e a comercialização. O fato de a aplicação de 1-MCP ter contribuído para maior preservação da FP pode estar relacionada à inibição do efeito autocatalítico do etileno, que estimula enzimas hidrolítcas, como poligalacturonases, pectil metil esterases, Bgalactosidases e celulases (Pech et al., 1999; Brummell \& Harpster, 2001; Zanuzo, 2004).

Quando se comparou a coloração dos caquis tratados e nãotratados com 1-MCP, não houve diferença significativa. Esperava-se que a aplicação de 1-MCP retardasse a velocidade de evolução de coloração avermelhada que, segundo Girardi et al. (2003), é afetada pelo etileno. Isso não ocorreu. A evolução da cor foi semelhante em caquis tratados e não-tratados com 1-MCP.

\section{Efeito da inibição do etileno em AR}

Ao analisar os frutos mantidos em ar refrigerado (AR), nenhum benefício significativo foi obtido, ou seja, tanto frutos tratados como não-tratados apresentaram coloração e FP semelhantes (Tabela 2). Porém, quando esses frutos foram mantidos em temperatura de $18 \pm 3^{\circ} \mathrm{C}$, buscando estudar a vida de prateleira, a inibição da ação do etileno mostrou-se eficiente, reduzindo a velocidade de perda da FP. Assim, por exemplo, os frutos tratados com 1-MCP apresentam 30,39 N e os nãotratados apresentaram-se completamente amolecidos. Vários trabalhos 
TABELA 2 - Qualidade de caquis 'Fuyu' armazenados sob refrigeração $\left(0 \pm 1^{\circ} \mathrm{C}\right)$, avaliados na colheita, aos 45 dias de armazenamento refrigerado e mais 3 dias a $18 \pm 3^{\circ} \mathrm{C}$ após a retirada da câmara.

\begin{tabular}{|c|c|c|c|}
\hline \multirow[t]{2}{*}{ Parâmetros } & \multirow{2}{*}{$\begin{array}{l}\text { Aplicação de } \\
1-M C P\end{array}$} & \multicolumn{2}{|c|}{ Períodos de análise } \\
\hline & & Colheita & $45+3$ dias \\
\hline \multirow[t]{2}{*}{ Firmeza de Polpa (N) } & Controle & $82,6 \mathrm{aA}^{*}$ & $0 \mathrm{aB}$ \\
\hline & 1-MCP & $82,6 \mathrm{aA}$ & $30,3 \mathrm{bB}$ \\
\hline \multirow[t]{2}{*}{ Cor "a" } & Controle & $34,6 \mathrm{aA}$ & $15,1 \mathrm{aB}$ \\
\hline & 1-MCP & $34,6 \mathrm{aA}$ & $20,9 \mathrm{aB}$ \\
\hline \multirow[t]{2}{*}{ Cor "b" } & Controle & $49,7 \mathrm{~A}$ & $21,7 \mathrm{aB}$ \\
\hline & 1-MCP & $49,7 \mathrm{~A}$ & $26,0 \mathrm{aB}$ \\
\hline \multirow[t]{2}{*}{ Cor "L" } & Controle & $56,3 \mathrm{aA}$ & $37,1 \mathrm{aB}$ \\
\hline & 1-MCP & $56,3 \mathrm{aA}$ & $40,6 \mathrm{aB}$ \\
\hline \multirow[t]{2}{*}{ Ângulo Hue } & Controle & $54,6 \mathrm{aA}$ & $55,2 \mathrm{aA}$ \\
\hline & 1-MCP & $54,6 \mathrm{aA}$ & $52,2 \mathrm{aA}$ \\
\hline \multirow[t]{2}{*}{ Escurecimento da epiderme (\%) } & Controle & 0 & 55,5 \\
\hline & 1-MCP & 0 & 44,4 \\
\hline
\end{tabular}

*Médias seguidas pela mesma letra, minúscula quando na mesma coluna e maiúscula entre colunas, não diferem estatisticamente, pelo teste de Tukey, a 5\% de probabilidade, para cada parâmetro avaliado.

(Brackmann et al., 2003; Ferri et al., 2002a), que analisaram caquis em AR, descreveram que os principais problemas detectados são a diminuição da FP e o aumento do escurecimento epidérmico.

Porém, se os frutos são mantidos em refrigeração, há rápida perda da firmeza de polpa. Esse comportamento foi verificado neste trabalho. O 1-MCP reduziu as perdas de FP, ou seja, três dias após em AR ainda mantinham FP maior que $20 \mathrm{~N}$, que é considerado como valor mínimo para comercialização e consumo de caqui 'Fuyu'.

No que concerne ao escurecimento, o 1-MCP não controlou este distúrbio. Frutos tratados e não-tratados com 1-MCP apresentaram mais de $60 \%$ de escurecimento da epiderme.

Embora as causas desse distúrbio não tenham sido esclarecidas, acredita-se que estejam associadas à formação de microfissuras na epiderme dos frutos, o que facilitaria a oxidação e o crescimento de microorganismos saprófitas. Desequilíbrios nutricionais, excesso de umidade no pomar e colheita tardia têm sido citados como prováveis indutores da formação de microfissuras (Gonçalves, 2003). Baseando-se nessas hipóteses, alguns trabalhos têm empregado suplementação mineral (Ferri et al., 2002b) e retardadores da maturação (Ben-Arie et al., 1996) para evitar esse problema. Os resultados não são conclusivos e, em alguns casos, não validados em trabalhos subseqüentes.

Dessa maneira, verificou-se que, para caquis 'Fuyu', conservados em AR, o 1-MCP teve efeito na maior preservação da firmeza de polpa sem afetar a coloração e a ocorrência do escurecimento.

Utilização de atmosfera modificada (AM) no armazenamento de caquis 'Fuyu'

Quando se associaram embalagens PEBD ao AR, preveniu-se a ocorrência de escurecimento epidérmico (Tabela 3). Além disso, a FP manteve-se acima de $60 \mathrm{~N}$ após quatro dias de retirada da câmara fria. Também ao analisarem-se os frutos após 48 dias de AM, mais 10 dias na temperatura ambiente $\left(18^{\circ} \mathrm{C}\right)$, a FP apresentou-se elevada, com valores de 48,1 N para frutos sem aplicação de 1-MCP e 65,73 $\mathrm{N}$ para frutos com 1-MCP. Esses dados demonstram o efeito benéfico da AM na preservação da FP e na prevenção do EE. O inibidor da ação do etileno, nesse caso à semelhança do AR, contribui apenas para maior firmeza de polpa. $\mathrm{O}$ efeito da AM na preservação da FP e na prevenção do EE foi semelhante ao observado por Brackmann et al. (2003), empregando AC, e por Ferri et al. (2002b), empregando AM.

\section{CONCLUSÕES}

1. A inibição da ação do etileno, com 1-MCP, preservou a FP, porém não reduziu a ocorrência de escurecimento epidérmico.

2. O emprego de atmosfera modificada prolonga o período de armazenamento por 48 dias, mais 10 dias de comercialização simulada $\left(18^{\circ} \mathrm{C}\right)$ do caqui 'Fuyu', mantendo a firmeza de polpa adequada e prevenindo a ocorrência de escurecimento epidérmico .

TABELA 3 - Qualidade de caquis 'Fuyu' armazenados sob refrigeração e acondicionados em atmosfera modificada, avaliados na colheita, aos 48 dias de câmaras, mais 4 dias e 10 dias em temperatura ambiente $\left(18 \pm 3^{\circ} \mathrm{C}\right)$.

\begin{tabular}{|c|c|c|c|c|}
\hline \multirow[t]{2}{*}{ Parâmetros } & \multirow{2}{*}{$\begin{array}{l}\text { Aplicação de 1- } \\
\text { MCP }\end{array}$} & \multicolumn{3}{|c|}{ Períodos de avaliação } \\
\hline & & Colheita & 7 dias & 14 dias \\
\hline \multirow[t]{2}{*}{ Firmeza de Polpa (N) } & Controle & $82,6 \mathrm{aA} *$ & $75,3 \mathrm{aA}$ & $32,6 \mathrm{aB}$ \\
\hline & 1-MCP & $85,8 \mathrm{aA}$ & $65,2 \mathrm{aAB}$ & $47,9 \mathrm{aB}$ \\
\hline \multirow[t]{2}{*}{ Cor "a" } & Controle & $34,6 \mathrm{aA}$ & $37,7 \mathrm{aA}$ & $36,6 \mathrm{aA}$ \\
\hline & 1-MCP & $36,1 \mathrm{aA}$ & $37,3 \mathrm{aA}$ & $42,6 \mathrm{aA}$ \\
\hline \multirow[t]{2}{*}{ Cor "b" } & Controle & $49,1 \mathrm{aA}$ & $49,9 \mathrm{aA}$ & $40,6 \mathrm{aA}$ \\
\hline & $1-\mathrm{MCP}$ & $49,3 \mathrm{aA}$ & $50,7 \mathrm{aA}$ & $50,4 \mathrm{aA}$ \\
\hline \multirow[t]{2}{*}{ Cor "L" } & Controle & $56,3 \mathrm{aA}$ & $54,7 \mathrm{aAB}$ & $52,2 \mathrm{aB}$ \\
\hline & 1-MCP & $55,8 \mathrm{aA}$ & $56,6 \mathrm{aA}$ & $53,5 \mathrm{aA}$ \\
\hline \multirow[t]{2}{*}{ Ângulo Hue } & Controle & $54,6 \mathrm{aA}$ & $52,9 \mathrm{aA}$ & $47,7 \mathrm{aA}$ \\
\hline & 1-МCP & $53,7 \mathrm{aA}$ & $53,8 \mathrm{aA}$ & $47,8 \mathrm{aA}$ \\
\hline \multirow[t]{2}{*}{ Escurecimento da epiderme (\%) } & Controle & 0 & 0 & 0 \\
\hline & 1-MCP & 0 & 0 & 0 \\
\hline
\end{tabular}

*Médias seguidas pela mesma letra, minúscula quando na mesma coluna e maiúscula entre colunas, não diferem estatisticamente, pelo teste de Tukey, a 5\% de probabilidade, para cada parâmetro avaliado. 


\section{REFERÊNCIAS}

ANTONIOLLI, L.R.; CASTRO, P.R. de C. e; KLUGE, R.A.; FILHO, J.A.S. Utilização de embalagem de polietileno na conservação de caquis 'Giombo' durante o armazenamento refrigerado. Revista Brasileira de Fruticultura, Jaboticabal, v. 25, n.1, p. 77 - 80, 2003.

BEN-ARIE, R.; SAKS, Y.; SONEGO, L.; FRANK, A. Cell wall metabolism in gibberellin-treated persimmon fruits. Plant Growth Regulation, Holanda, v. 19, p. 25-33, 1996.

BRACKMANN, A.; FREITAS, S.T.; MELLO, A.M. de; STEFFENS, C.A. Aplicação de 1-MCP em caqui ‘Quioto’ armazenado sob refrigeração e atmosfera controlada. Revista Brasileira de Fruticultura, Jaboticabal, v. 25, n.1, p. 42-44, 2003.

BRACKMANN, A.; DONAZZOLO, J. Armazenagem de caqui. In.: ENFRUTE - Encontro Nacional sobre Fruticultura de Clima Temperado, 3., 2000, Fraiburgo. Anais ... Videira: EPAGRI, p.99-102, 2000.

BRACKMANN, A.; MAZARO, S.M.; SAQUET, A.A. Frigoconservação de caquis (Diospyrus kaki, L.) das cultivares Fuyu e Rama forte. Ciência Rural, Santa Maria, v. 27, n.4, p. 561 - 565, 1997.

BRACKMANN, A.; SAQUET, A.A. Efeito da temperatura e condições de atmosfera controlada sobre a conservação de caqui (Diospyrus kaki, L.). Ciência Rural, Santa Maria, v. 25, n.2, p. 215-218, 1995.

BRUMMELL D.A.; HARPSTER, M.H. Cell wall metabolism in fruit softening and quality and its manipulation in transgenic plants. Plant Molecular Biology, Dordrecht, v.47, p. 311-340, 2001.

CORRENT, A. R. 1-Metilciclopropano na conservação de maçãs 'Royal Gala'e 'Fuji'. 2003. 69f. Dissertação (Mestrado em Ciência e Tecnologia Agroindustrial)- Universidade Federal de Pelotas, Pelotas, 2003.

DONAZZOLO, J.; BRACKMANN, A. Armazenamento de caqui (Diospyrus kaki, L.) cv. Quioto, em atmosfera controlada. Revista Brasileira de Fruticultura, Jaboticabal, v. 24, n.2, p. 323-327, 2002.

FERRI, V.C.; RINALDI, M.M.; DANIELI, R.; LUCHETA, L.; ROMBALDI, C.V. Controle da maturação de caquis 'Fuyu', com uso de aminoethoxivinilglicina e ácido giberélico. Revista Brasileira de Fruticultura, Jaboticabal, v. 24, n.2, p. 344 - 347, 2002a.
FERRI, V.C.; RINALDI, M.M.; LUCHETA,L.; ROMBALDI, C.V. Qualidade de caquis Fuyu tratados com cálcio em pré-colheita e armazenados sob atmosfera modificada. Revista Brasileira de Fruticultura, Jaboticabal, v. 24, n.2, p. 385 - 388, 2002 b.

GIRARDI, C.L.; PARUSSOLO, A.; DANIELI, R.; CORRENT, A.R.; ROMBALDI, C.V. Conservação de caqui (Diospyrus kaki, L.), cv. Fuyu, pela aplicação de 1-Metilciclopropeno. Revista Brasileira de Fruticultura, Jaboticabal, v. 25, n.1, p. 54-56, 2003.

GONÇALVES, E.D.; Variabilidade genética, conservação e ocorrência de escurecimento de caquis (Diospyrus kaki, L.) cv. Fuyu. 2003. $49 \mathrm{f}$. Tese (Doutorado em Fruticultura de Clima Temperado)- Universidade Federal de Pelotas, Pelotas, 2003.

GORINI, F.L.; TESTONI, A. Raccolta, conservazione e trasformazione dei frutti di kaki. Analli dell'Istituto Sperimentale per la Valorizzazione Tecnológica dei Prodotti Agricoli, Milano, v. 19, p. 249-258, 1988.

JOÃO, P.L.; ROSA, J.I. da; FERRI, V.C.; MARTINELLO, M.D.; Levantamento da Fruticultura Comercial do Rio Grande do Sul. Porto Alegre: EMATER/RS-ASCAR, 2002. (Série Realidade Rural, 28).

NEVES, L.C.; CORRENT, A.; MARINI, L.; LUCCHETTA, L.; ZANUZO, M. R.; GONÇALVES, E.D.; ZANATTA, J.; CANTILLANO, F.R.; ROMBALDI, C.V. Atmosfera modificada e 1-Metilciclopropeno na conservação pós-colheita de kiwis cv. Bruno. Revista Brasileira de Fruticultura, Jaboticabal, v. 25, n.3, p. 390 - 393, 2003.

PECH, J.C.; GUIS, M.; BOTONDI, R.; AYUB, R.; BOUZAYEN, M.; LELIEVRE, J.M.; EL YAHYAOUI, F.; LATCHE, A. 1999: Ethylenedependent and ethylene-independent pathways in a climacteric fruit, the melon. In: Kanellis, A.K.; et al. (Ed.). Biology and biotechnology of the plant hormone ethylene II. Dordrecht: Kluwer Academic Publishers, 1999. p. 105-110.

SISLER, E.C. Ethylene binding components in plants. In: MATTOO, A. K.; SUTTLE, J. C. (Ed.). The plant hormone ethylene. Boca Raton: CRC Press, 1991, p.81-99.

ZANUZO, M. Etileno na transcrição de poligalacturonases em melões transformados geneticamente com o clone pAP4 antisense. 2004. 66f. Dissertação (Mestrado em Ciência e Tecnologia Agroindustrial)Universidade Federal de Pelotas, Pelotas, 2004. 